\title{
New Perspectives in the Pharmacologic Treatment of Primary Open-Angle Glaucoma: Pathogenesis and Patient Factors
}

\author{
Kevin A. Waschke*, B.Sc.
}

* To whom correspondence should be addressed: Faculty of Medicine, McGill University, 3655 Drummond St., Montreal, QC, Canada H3G 1 Y6

\section{INTRODUCTION}

Glaucoma, the most prevalent disease facing ophthalmologists today, is a collection of disorders characterized by progressive loss of visual fields due to optic nerve damage and, in most cases, elevation of intraocular pressure (IOP). They are the leading cause of blindness in the United States, affecting 1-2\% of individuals aged 60 and over. Because the progressive vision loss tends to be painless, patients often present with advanced disease (1). Early detection depends on routine eye examinations with IOP measurements (using tonometry), attention to the appearance of the optic disc, and visual field testing.

Classification of the glaucomas has important implications for treatment and prognosis. Whereas primary glaucoma is unrelated to other ocular disease, secondary glaucoma occurs as a consequence of other ocular abnormalities or pathology. Glaucomas are also identified by anterior chamber tissue alterations (for example, congenital neovascular glaucoma), by accumulation of abnormal substances (including macrophages, degraded lens protein, tumor cells, altered blood cells, and melanin pigment) in the anterior chamber angle, and by the anatomical configuration of the anterior chamber angle (open- or closed-angle glaucoma) (2). If greater than 20 degrees, the anterior chamber angle--the angle formed by the anterior surface of the iris, the face of the ciliary body, the internal surface of the trabecular meshwork, and the posterior surface of the cornea--is, by definition, open. Closed-angle glaucoma occurs as a result of increased pupillary resistance to aqueous humor outflow, and may be acute (with increased IOP) or chronic (with normal IOP) (3).

Accounting for $75 \%$ of all glaucomas, primary open-angle glaucoma (POAG) is the most common type. In POAG, the anterior chamber angle is open, permitting free access of aqueous humor to the structures in the angle responsible for fluid drainage. It typically presents as asymptomatic, progressive loss of peripheral and paracentral visual fields leading to blindness if untreated. Risk factors include advanced age, male gender, black race, high IOP, family history of open-angle glaucoma, myopia, stress, adult-onset diabetes mellitus, thyroid disorders, and chronic systemic disease $(4,5)$. Decreased lean body mass and history of cataracts have a weaker association (6), and studies on the role of vascular factors remain contradictory (7), although an increased prevalence of vasospastic disorders and systemic orthostatic hypotension among glaucoma patients has been shown (8-11). 
The pathogenesis of POAG has not yet been elucidated. Advances at the molecular level have not thus far translated into a clear understanding of ocular receptors and the mechanism by which anti-glaucoma drugs, such as B-blockers, inhibit aqueous humor formation. Similarly, current animal models do not provide adequate simulations of human glaucoma: ethacrynic acid has been shown to increase outflow of aqueous humor in monkeys but has failed in human trials (12). Ongoing development of new drug classes, refinement of older drug classes, changing theories of pathogenesis, improvement in laser and surgical procedures (1315), and increasing knowledge and concern about side effects and patient compliance necessitate the constant re-evaluation of current and potential pharmacologic treatment of POAG.

\section{AQUEOUS HUMOR PRODUCTION AND DRAINAGE}

Aqueous humor is a clear fluid high in ascorbic acid and low in protein that is continually produced by the ciliary processes of the non-pigmented epithelium of the ciliary body at a rate of approximately $2.5 \mu \mathrm{L} / \mathrm{min}$. It is responsible for maintaining the normal pressure and volume of the eye and providing nutrition to avascular eye tissue, including the lens, cornea, and vitreous body. The production of aqueous humor depends on three physiologic processes: diffusion, ultrafiltration, and secretion. Diffusion involves the movement of substances across cell membranes along local transmembrane concentration gradients. Ultrafiltration depends on the osmotic and hydrostatic pressure differences between the plasma in the capillaries of the ciliary processes and the aqueous humor in the posterior chamber. Secretion occurs by transepithelial fluid movement linked to $\mathrm{Na}^{+}, \mathrm{K}^{+}$-ATPase and carbonic anhydrase enzymes in the non-pigmented ciliary epithelium. Varying with the diurnal cycle of epinephrine release from the adrenal glands, secretion is highest during the day and lowest at night $(3,16)$.

Once produced by the ciliary body, the aqueous humor travels from the posterior chamber of the eye into the anterior chamber via the pupil. The first point of resistance to outflow occurs at the pupil by contact of the iris with the anterior surface of the lens. The second and major site of resistance lies between the anterior chamber and the venous circulation in the draining structures of the anterior angle. Approximately $85 \%$ of the fluid passes through the corneoscleral trabecular meshwork into Schlemm's canal and drains into the episcleral aqueous veins. The remaining $15 \%$ passes through the uveoscleral vascular system which then communicates with the systemic venous circulation (17).

The aqueous fluid flows from the trabecular meshwork into Schlemm's canal, a large, endothelium-lined drainage vessel, and enters the venous circulation via 25-35 collector channels. The trabecular meshwork, composed of lamellated sheets bordering Schlemm's canal, may be divided into two parts. The most anterior, non-filtering portion has no contact with Schlemm's canal. The filtering portion, which covers the inner wall of Schlemm's canal, consists of three layers (from outward to inward): the cribiform layer, the corneoscleral meshwork, and the uveoscleral meshwork. The cribiform layer consists of layers of elongated, fibroblast-like cells embedded in a homogeneous extracellular material. The acellular spaces seen by electron microscopy may represent channels for the flow of aqueous fluid toward Schlemm's canal. The corneoscleral meshwork, the main layer of the trabecular meshwork, is composed of flat, interlacing beams, each of which contains a central core of collagen, ground substance, and elastic fibers lined by endothelium and supported by a basement membrane. The uveoscleral meshwork consists of similarly composed but irregularly arranged strands covered by a layer of endothelial cells.

The cells of the trabecular meshwork have several functions. They produce glycosaminoglycans (GAGs), extracellular glycoproteins, and fibrillar material. They are also highly phagocytic, capable of removing particles, pigment granules, cellular debris, red blood cells, iron, and bacteria from the aqueous humor, thereby preventing obstruction of outflow drainage. In certain cases of secondary open-angle glaucoma, this phagocytic function is overwhelmed, leading to blockage of the trabecular meshwork.

A number of factors play a role in the normal eye's resistance to outflow at the anterior chamber angle (17). 
Changes in IOP produce changes in the arrangement of trabecular fibers, such that the balance between inflow and outflow of aqueous humor remains constant. The ciliary muscle also affects the arrangement of the trabecular meshwork by virtue of its anatomical attachment to the scleral spur: contraction alters the orientation of the meshwork to produce an increased corneoscleral, and perhaps also uveoscleral, outflow. Studies have shown that increasing hyalinization of trabecular columns with age leads to increased resistance to outflow, but does not lead to pathologically high IOP levels due to the concomitant, age-related decrease in aqueous humor secretion (18). Approximately $1 / 3$ of the population have been shown to exhibit a significant increase in IOP after topical or systemic administration of corticosteroids, $3 \%$ of whom develop "cortisone glaucoma". Although the pathogenesis of this condition is not clear, it has been suggested that corticosteroids may affect the polymerization of acid mucopolysaccharides within the trabecular meshwork. Neurogenic regulation of IOP via the rich plexus of nerve fibers and endings within the meshwork has also been postulated to affect IOP and seems to be supported by microelectrode studies of cat hypothalami (17). Any increase in episcleral venous pressure can lead to increased IOP secondary to decreased aqueous drainage, and is, in fact, the cause of one type of secondary open-angle glaucoma.

\section{THEORIES OF PATHOGENESIS}

The defect responsible for increased resistance to aqueous humor outflow in POAG is less straightforward and less discernible than that in secondary glaucoma. In fact, the actual location of the postulated defect remains controversial. Although hypersecretion of aqueous humor has been reported in exceptional cases, it is generally not believed to be a factor in the pathogenesis of POAG. It is almost certain that the major part of outflow resistance is not located beyond Schlemm's canal. Some have postulated the defect to be a decrease in vacuolization of inner wall endothelium in Schlemm's canal (which would reduce the ability of Schlemm's canal to collect drainage fluid), but studies have failed to show any difference between normal and affected eyes. Furthermore, there is no consensus as to whether the collapse of Schlemm's canal occasionally seen in sections is the primary cause of resistance to outflow or is a secondary effect. Studies of trabeculoplasty specimens showing an abnormal accumulation of protein in Schlemm's canal are of questionable value due to the potential for protein leakage and damage during surgery $(2,3,19)$.

Most data suggest that the pathogenesis of POAG is related to a defect in the trabecular meshwork. There is a loss of the inner meshwork trabecular cells, thought to occur due to chronic phagocytic demand on the cells. Sheath-derived (SD) plaques, originating from the sheath of elastic-like fiber beneath the endothelial lining of Schlemm's canal, have been shown to be more abundant in the cribiform layer of glaucomatous eyes than in the eyes of normal age-matched controls. Some studies indicate that there may be an abnormal hyalinization of the trabecular meshwork beams. In both cases, a subtle narrowing of collector channels might lead to a large increase in outflow resistance, leading to pathologically increased IOP in some glaucomatous eyes $(2,3,19)$.

There has been a long-standing belief that the mechanism of damage in glaucomatous eyes is related to IOP. Indeed, the persistence of this belief is reflected in the current treatment available, which aims to reduce IOP to somewhat arbitrary target pressures (1). Although there is no evidence to suggest that increased IOP causes the loss of ganglion cells and nerve fiber substance in the retina directly, there is a very strong association between glaucoma and IOP above the normal range of 10-22 $\mathrm{mm} \mathrm{Hg}$. It has been shown that the higher the IOP, the greater the probability of developing glaucoma: $42 \%$ of patients with IOP $>30 \mathrm{~mm} \mathrm{Hg}$ develop glaucoma within 5 years (20). Subjects with pressures ranging from 16-19 $\mathrm{mm} \mathrm{Hg}$ are at 1.7 times the risk of patients with lower pressures for developing glaucomatous change, and the risk increases 10.5 -fold when the pressure exceeds $24 \mathrm{~mm} \mathrm{Hg}$ (21). Primate eyes exposed to increased IOP for extended periods exhibit neuropathy that is clinically and histologically indistinguishable from human POAG. In addition, the eye with higher IOP in patients with normal-tension glaucoma always exhibits greater visual field loss. However, there is no critical target pressure beyond which IOP can be reduced to prevent further progressive degeneration of the optic disk and nerve bundle damage. 
Current research appears to indicate that the damaging effect of IOP occurs at the level of the lamina cribrosa. This structure consists of ten lamellar sheets, the aligned pores of which form channels for optic nerve bundles containing the axons of retinal ganglion cells. The lamina cribrosa provides structural support for the axons and contains the vascular supply for that part of the optic nerve. In vitro studies have shown that high pressure can distort or collapse the lamina cribrosa, which could lead to retinal ganglion cell axon damage. In fact, the regions of the lamina cribrosa with thicker septae (and thus possibly greater resistance to the effects of mechanical deformation) have been shown to correspond to retinal nerve fibers supplying portions of the visual field relatively spared from damage. From a theoretical point of view, this concept may explain why patients with axial myopia (higher axial length than emmetropic eyes) have been shown to be at a higher risk for glaucoma. By Laplace's equation, the larger radius leads to an elevated wall stress with an increase in the potentially detrimental force on the optic nerve. Other studies have shown that experimentally-induced glaucoma causes displacement of the optic nerve head, which may cause disturbances in axonal transport. Changes in the extracellular matrix may also be associated with increased IOP, potentially resulting as well in altered delivery of nutrients to axons. It would seem, therefore, that individual variations in the lamina cribrosa may be related to patients' susceptibility to the effects of increased IOP (22-24).

This collection of evidence supporting a pressure-related mechanism of damage does not explain why some patients with "normal" IOP develop damage, while others with ocular hypertension do not. Studies showing an increased prevalence of diabetes mellitus, hypertension, and migraines in glaucoma patients--diseases with well-known microvascular or angiogenic abnormalities--have lent support to the concept that both vasogenic and pressure-related mechanisms may be involved in optic nerve damage. The current hypothesis is that vascular dysregulation may lead to a temporary or permanent decrease in optic nerve head perfusion, which may contribute to the development of glaucomatous damage in some patients. Unfortunately, researchers do not currently have the ability to quantify ocular perfusion in the optic nerve head with sufficient accuracy to yield reliable results in clinical trials. While studies with fluorescein angiography only depict the superficial layer of blood vessels arising from the retina, laser Doppler velocimetry has shown some promise (22-24).

\section{PHARMACOLOGIC TREATMENT}

The therapy for glaucoma patients is highly individualized, but typically begins with the use of topical agents, usually ß-blockers. If the pressure reduction is not sufficient, systemic carbonic anhydrase inhibitors are used to decrease aqueous humor production, followed by laser trabeculoplasty and filtration surgery if medical therapy fails. Cases of severe glaucoma (defined as an IOP of greater than $30 \mathrm{~mm} \mathrm{Hg}$ ) should be treated immediately on presentation with trabeculoplasty (25).

The chronic asymptomatic nature of POAG presents the physician with a significant problem of patient noncompliance. In the face of the complexity of therapeutic regimens, the chronic use of multiple medications, the need for frequent daily administration, the lack of noticeable beneficial effect, the presence of side effects, and financial cost, patients often do not take their medication consistently. One study using a pilocarpine eyedrop monitor demonstrated that subjects took a mean of $76 \%$ of their eyedrops, but reported taking $97 \%$ (3). Patients often take their medication as prescribed on the day of a visit to the doctor, but when asked, admit to not taking their medication on a regular basis. Ability to administer eyedrops and understanding of the disease and treatment are important factors. In one study, $13 \%$ of patients experienced in the use of topical eyedrops were unable to instill topical medications into both eyes successfully even after several attempts (26).

To ensure compliance, counselling and education regarding the disease process and its therapy are vital. A physician or nurse should watch the patient instill drops and demonstrate how to apply pressure over the lower puncta and canaliculi in order to minimize systemic side effects (16). The drug regimen should be integrated into the patient's daily routine. In some cases, switching the currently used drug for a "new" drug of the same class with similar effects has been shown to improve compliance (27). Bottles recording drop 
administration can help physicians to monitor compliance, and patients to remember to take their medications; card charts with an eyedrop administration schedule can aid patients with complex regimens (16). Insufficient physician attention to patient compliance often leads to progressive optic nerve damage and visual field loss and a need for laser therapy or filtering surgery; unfortunately, physicians often make the erroneous assumption that failure of medical therapy is a result of low drug effectiveness.

The need for personalization of glaucoma therapy cannot be overemphasized. Choice of an appropriate pharmacologic agent must take into account coexistent medical conditions and risk factors in order to avoid the development of adverse ocular or systemic side effects, which can produce significant complications and decrease compliance. As the underlying pathogenetic defect is not yet fully understood, there is no therapy available to reverse POAG. It is, therefore, important to recall that success of glaucoma therapy is not judged by the degree of IOP reduction alone, since there is wide individual variation in the range of pressures necessary to produce glaucomatous damage. Thus, measurements of IOP provide no indication of drug efficacy without frequent examination of the optic nerve head and visual fields, careful evaluation of the presence and severity of side effects, and assessment of patient compliance.

\section{B-Blockers}

ß-adrenergic antagonists, which were serendipitously found to reduce IOP over 25 years ago during the use of propranolol to reduce systemic hypertension, are the initial therapy of choice in POAG (28). Timolol, a nonspecific B-blocker, quickly became the gold standard for medical therapy of glaucoma, particularly POAG. There are presently five $\beta$-blockers in use (timolol, betaxolol, levobunolol, carteolol, and metipranolol), all of which decrease IOP by reducing aqueous humor production via a predominantly $\beta_{2}$-adrenergic receptor mechanism in the ciliary epithelium. By antagonizing epinephrine, timolol blocks potential increases in aqueous humor production. As would be expected, application of ß-blockers at night has very little effect on aqueous humor production, since circulating epinephrine levels are already low. Morphologic changes in the non-pigmented ciliary epithelium have also been observed following timolol administration, suggesting the cessation of cellular pumping action (29).

The typical half-life of B-blockers following oral administration ranges from 3-12 hours. Topical B-blockers, on the other hand, have been shown to be effective for 12-24 hours due to significant binding to melanin pigment in the eye; this is one reason for the greater challenge that glaucoma control represents in patients with heavily pigmented eyes. Once therapy is stopped, the ocular hypotensive effect of $\beta$-blockers persists for several weeks, much longer than would be expected from the half-life. It is thought that this persistence of effect may be caused by down-regulation of adrenergic receptors in the ciliary epithelium.

The main differences among the $\beta$-blockers lie in their local tolerability and their production of systemic side effects. Indeed, B-blockers are favored over other drug classes largely due to their extended duration of action and low level of ocular side effects. In terms of systemic effects, the distinction between selectivity and nonselectivity is crucial, because up to $80 \%$ of a topically administered drug can enter the general circulation by drainage via the nasolacrimal duct and absorption by the nasal mucosa, at concentrations sufficiently high to produce side effects. The non-selective $\beta$-blockers (timolol, levobunalol, and metipranalol) can produce suppression of heart rate and myocardial contractility during exercise and cause alterations in cardiac rate and rhythm, such as sinus bradycardia. They can also have a negative effect on lipid metabolism, producing an elevation in serum triglycerides and a decreased high-density lipoprotein level. One study estimates that their negative effect on the lipid profile potentially constitutes a $17 \%$ increased risk of myocardial infarction. Central nervous system effects, such as depression, altered mental states, and impotence, have also been reported with the use of ocular $\beta$-blockers, and it has been suggested that the single greatest risk factor for falls in elderly patients with glaucoma is the use of topical $\beta$-blockers $(30,31)$.

The side effect of non-specific $\beta$-blockers that has generated the most concern, particularly with timolol, 
involves respiratory function. Non-selective $\beta$-blockers are contraindicated in patients with bronchospastic disease. Pulmonary function tests have been shown to worsen after the administration of a single drop of timolol in patients with asthma, chronic obstructive pulmonary disease, and chronic bronchitis (32). This exacerbation of underlying pulmonary disease is especially important when one considers the high prevalence of both glaucoma and airway diseases in the elderly. A recent study in Britain indicates that the seriousness of this complication may be dangerously underestimated, as more than $25 \%$ of the study population had undiagnosed obstructive airway disease as revealed by improved respiratory function on change of therapy (33). Although timolol's mode of application and poor lipid solubility produce cerebral and systemic concentrations that are low in comparison to systemic B-blockers such as propranolol, other side effects continue to generate concern. Timolol may mask diabetes mellitus and contribute to the polypharmacy of old age, enhancing the potential for serious drug interactions. It is also possible that central nervous system effects--including disorientation, hallucinations, depression, fatigue, anxiety, emotional lability, and memory impairment--have been underreported and attributed instead to systemic or cerebrovascular disease. Timolol also consistently reduces heart rate and, like other non-specific B-blockers, worsens congestive heart failure. Other reported side effects include syncope, bradyarrythmias, heart block, fibrillation, and myocardial infarction, as well as impotence, rashes, diarrhea, male-pattern baldness, and reduction of high-density lipoproteins (28).

Levobunolol and metipranolol are the two other principal non-selective $\beta$-blockers. Levobunolol has a very potent effect on IOP, but shares timolol's effect on systemic B-adrenergic receptors. It has slightly more ocular side effects than timolol and should be avoided in patients with cardiovascular or respiratory problems. Its metabolite dihydrolevobunolol is active and has a half-life of 7 hours, making levobunolol useful for oncedaily therapy, like timolol. Although clinical experience with metipranolol is limited, it has an oculohypotensive effect comparable to that of timolol and levobunolol, without cardiac or pulmonary side effects other than a slightly decreased pulse (1).

The presence of side effects has inspired the search for better ß-blockers. Carteolol, for example, has partial agonist activity; this intrinsic sympathomimetic activity (ISA) is thought to cause fewer alterations in lipid profile and heart rate, an important consideration in patients with high blood pressure. The ISA of carteolol may also improve retinal blood flow. Its effect on IOP is similar to that of timolol, and its active metabolite 8hydroxy-carteolol has a half-life 2-3 times that of carteolol, which may increase bioavailability and duration of action. Of all the ocular B-blockers, certeolol is probably the most well-tolerated, although its long-term effects remain to be studied $(28,34)$.

Betaxolol, the only $ß_{1}$-selective blocker, has shown, after seven years of use, no apparent systemic side effects. There have been no changes in pulmonary function observed in patients with chronic obstructive pulmonary disease, asthma, and chronic bronchitis. Betaxolol is similar in ocular hypotensive effect to timolol, although some studies have shown it to be slightly less effective (28). Some authors recommend the use of betaxolol over timolol due to the absence of side effects and to the fact that a statistically significant difference in IOP induced by a drug may not produce clinically significant effects. However, it has been suggested that the large number of reported side effects of timolol may only reflect the long history of the use of timolol as compared to other IOP-lowering drugs. Long-term studies of betaxolol are crucial, as drugs can produce delayed effects and allergic responses when taken chronically on a daily basis.

The choice of ß-blockers depends on a personalized assessment of potential adverse systemic effects, since each of the B-blockers is essentially equally effective. Although IOP-lowering effect is not a measure of drug efficacy on its own, numerous studies continue to compare ocular hypotensive effects, using timolol as the gold standard. With the advent of new drug classes

and drug delivery methods, as well as advances in laser and filtration surgery, the use of timolol and other $\beta$ blockers is being challenged. 
Exemplified by pilocarpine, these drugs act by mimicking the action of acetylcholine on the iris sphincter and ciliary muscle. The contraction induced by pilocarpine produces a decreased outflow resistance in the trabecular meshwork and, hence, a decreased IOP. Pilocarpine, which is available as eye drops, as an ointment, and as an ocular insert similar to a contact lens, covers the range of current ocular drug delivery methods. An unavoidable side effect of para- sympathomimetics is a decrease in the amount of light entering the eye; they are therefore contraindicated in patients with cataracts, whose vision is already compromised. Vision may become blurry intermittently throughout the day, especially in younger patients, and headaches for the first few days after onset of treatment are common. Stronger miotics have a greater effect on IOP but are also occasionally associated with side effects, such as diaphoresis, nausea, and stomach cramps. There is also a possibility of cataract formation and retinal detachment (35).

\section{Adrenergic Agonists}

Topical adrenergic agonists, such as epinephrine and dipivefrine, function by facilitating uveoscleral and trabecular outflow. These drugs commonly cause stinging, tearing, and burning when first placed on the eye, leaving the eye bloodshot for several hours after application. Furthermore, they can produce allergic reactions and should be used with caution in patients with high blood pressure, irregular heart beats, and rapid pulses, or in patients who are aphakic or pseudophakic $(31) .{ }_{2}{ }_{2}$-adrenergic agonists, like apraclonidine, cause a shortterm reduction in IOP, mainly by decreasing aqueous humor production. They have also been postulated to affect uveoscleral outflow. Side effects include conjunctival blanching, minimal mydriasis, and eyelid retraction. Their long-term effects are still under investigation (36).

\section{Carbonic Anhydrase Inhibitors}

Although they have an extremely beneficial effect on IOP, oral systemic carbonic anhydrase inhibitors, such as acetazolamide, have severe systemic side effects and therefore have been used only after all other medical options have been exhausted. The carbonic anhydrase inhibitors act by inhibiting $\mathrm{HCO}_{3}{ }^{-}$movement into the posterior chamber and hence that of $\mathrm{Na}^{+}$and $\mathrm{H}_{2} \mathrm{O}$, with resultant inhibition of aqueous humor formation. It is interesting to note that cardiac glycosides have been shown to inhibit aqueous humor production in a similar manner by inhibiting $\mathrm{Na}^{+}, \mathrm{K}^{+}$-ATPase, but are not used clinically due to ocular toxicity (16). Side effects are severe for several reasons: inhibition of at least $99 \%$ of carbonic anhydrase activity must occur before aqueous humor production is significantly depressed; carbonic anhydrase is present in virtually every tissue; and high protein binding (95\%) necessitates large doses. Patient non-compliance is approximately $50 \%$ because of the severity of side effects (33): tingling in the hands and feet, poor appetite and sense of taste, stomach upset, diarrhea, fatigue, decreased libido, and depression. Rarer effects include kidney stones, thrombocytopenia, aplastic anemia, and agranulocytosis. The large variation in plasma levels after the same dose is given in different individuals makes it difficult to optimize dosages and minimize side effects. Carbonic anhydrase inhibitors should be avoided in patients with allergies to sulfa drugs (11).

In the past, topical carbonic anhydrase inhibitors were unable to reduce IOP significantly due to poor corneal penetration. However, because of the potential for greatly reduced side effects, research persisted for over twenty years and finally yielded the new topical carbonic anhydrase inhibitor, dorzolamide (MK-507). One year of clinical testing has shown reductions in IOP of up to $26.2 \%$ and no significant side effects following 3 -times daily therapy with a $2 \%$ preparation (29). Relatively few minor ocular side effects, including transient stinging, burning, and tearing, have been reported.

\section{NEW DIRECTIONS}


New avenues of research may affect future pharmacologic therapy. Very little attention has been paid to the possibility that some drugs used to reduce IOP may have deleterious effects on visual function. For instance, it has been found that $B$-adrenergic blockers, the first-line therapy for glaucoma, reduce ocular blood flow in experimental animals, with potentially negative effects on the optic nerve. From a physiologic standpoint, decreasing the flow of aqueous humor is not an ideal approach for glaucoma therapy when one considers that this flow is essential for nutrition of avascular ocular tissues $(37,38)$. Although clinically-evident short-term damage in these tissues has not been shown, there is a reduction in the safety margin of nutritional supply levels. In addition, the reduction of aqueous humor flow through the vitreous body to the posterior chamber may limit diffusion and removal of eye solutes, whose accumulations may be toxic to the ganglion cell axons. The use of parasympathomimetics is also suboptimal since their induction of ciliary muscle contraction not only increases corneoscleral outflow but simultaneously reduces uveoscleral outflow (31).

The ubiquity of carbonic anhydrase throughout the body not only contributes to systemic effects of inhibition but may also result in deleterious effects on ocular tissues. Carbonic anhydrase activity in the retina and choroid, including superficial retinal vessels, may produce a direct adverse effect. The problems associated with carbonic anhydrase inhibitors illustrate the basic pharmacologic principle that antagonists, which rarely participate in normal physiological control mechanisms and tend to evolve as toxins in nature (e.g., curare and atropine), tend to produce more side effects than agonists, which function by augmenting normal processes. Furthermore, antagonists require high effector cell concentrations for activity. Carbonic anhydrase is crucial for the maintenance of normal corneal hydration and transparency, and thus its inhibition may render the tissue more vulnerable to stresses.

The finding that the susceptibility to optic nerve damage in glaucoma may be related to mechanical, vascular, and other factors has created the potential for future reorientation of pharmacologic and surgical glaucoma therapy. Research has already shown that dopamine antagonists may be used to reduce IOP and, as an independent effect, to increase ocular pulsatile blood flow $(39,40)$. Recent investigations with $\mathrm{Ca}^{2+}$-channel blockers have also shown an improvement in visual fields and reduction in progressive optic nerve damage in low-tension glaucoma patients, presumably due to an improvement of ocular circulation $(41,42)$.

Epidemiological studies have shown evidence of a bimodal population distribution of POAG in which the first group exhibited a vasospastic response in their fingers to cold, whereas the second had risk factors for arterial disease with disturbed coagulation and biochemical measurements. High- and low-tension glaucoma cases were shown to be equally distributed between the two groups (43). Thus, future elucidation of the mechanisms of optic nerve damage will likely support the current belief that ocular hypertensive therapy alone will only benefit a select group of glaucoma patients. For this reason, research into factors that can provide protection to the optic nerve is currently underway. Growth factors showing promise include nerve growth factor (NGF), which is involved in nerve regeneration by stimulating outgrowth of neurites, and fibroblast growth factor (FGF), which may increase retinal ganglion cell survival (31). Another new drug currently being studied is the prostaglandin $\mathrm{F}_{2 *}$ analog, latanoprost. This drug exerts its effect by enhancing uveoscleral outflow and produces a marked reduction in IOP. In clinical trials of once-daily therapy used concomitantly with timolol for up to 12 weeks, no clinically significant side effects were observed during treatment (44).

New approaches to the design of safer ophthalmic drugs aim to limit effects to the desired site of action. Currently under investigation is the enzymatic activation of pro-drugs by enzymes preferentially or exclusively found at the site of action, the ciliary body. Similarly, the so-called "soft drug" approach involves several strategies, the most effective of which has been the modification of an active metabolite to produce a drug pharmacodynamically identical to the original drug but easily inactivated by a single-step reaction occurring virtually everywhere in the body (usually hydrolysis). Thus, the desired effect is produced exclusively at or near the site of administration (45). 
Clearly, the practicing ophthalmologist has a wide variety of medications with which to treat patients suffering from POAG. Assessment of pharmacologic therapy should involve not only measurements of IOP but also attention to the optic disc, visual fields, side effects, and compliance. With the advent of new drug classes and the discovery that alternate mechanisms may be involved in the pathogenesis of glaucoma, medical treatment--along with laser therapy and filtering surgery--will no doubt continue to provide new and exciting options for the management of POAG.

\section{ACKNOWLEDGMENTS}

The author wishes to thank the following individuals for their critique of the manuscript: Dr. Carlos Alcocer, Dr. Robert Lewandowski, Dr. Jacquetta Trasler, and Dr. Moy Fong Chen.

\section{REFERENCES}

1. Albert DM. Principles and Practice of Ophthalmology. Philadelphia, PA: WB Saunders and Co.; 1994: 1342-1345, 1569-1577.

2. Spencer WH. Ophthalmic Pathology. New York: WB Saunders and Co.; 1986: 487-547.

3. Ritch R, Shields MB, Krupin T, eds. The Glaucomas. St. Louis, MO: CV Mosby and Co.; 1989: 199-218, 827.

4. Johnson DH, Brubaker RF. Glaucoma: An Overview. Mayo Clinic Proceedings 6(1): 59-67; 1986.

5. Klein BE, Klein R, Jensen SC. Open-angle glaucoma and older-onset diabetes. The Beaver Dam Eye Study. Ophthalmology 101(7): 1173-1177; 1994.

6. Leske MC, Connell AM, Wu SY, et al. Risk factors for open-angle glaucoma. The Barbados Eye Study, Archives of Ophthalmology 113(7): 918-924; 1995.

7. Carter CJ, Brooks DE, Doyle DL, Drance SM. Investigations into a vascular etiology for low tension glaucoma. Ophthalmology 97(1): 49-55; 1990.

8. Drance SM, et al. Studies of factors involved in the production of low tension glaucoma. Archives of Ophthalmology 89(6): 457-465; 1973.

9. Drance SM, Douglas GR, Wijsman K, et al. Response of blood flow to warm and cold in normal and low tension glaucoma patients. American Journal of Ophthalmology 105(1): 35-39; 1988.

10. Gasser P. Ocular vasospasm: a risk factor in the pathogenesis of low-tension glaucoma. International Ophthalmology 13(4): 282-290; 1989.

11. Phelps CD, Corbett JJ. Migraine and low-tension glaucoma: a case-control study. Inverstigational Ophthalmology \& Visual Science 26(8): 254-263, 1105-1108; 1985.

12. Friedlaender M, Bartlett J. Brave new world of ophthalmic drugs. Eyecare Technology Magazine; 1995.

13. The Glaucoma Laser Trial (GLT): 6. Treatment group differences in visual field changes. Glaucoma Laser Trial Research Group. American Journal of Ophthalmology 120(1): 10-22; 1995.

14. Migdal C. What is the appropriate treatment for patients with primary open-angle glaucoma: medicine, laser or primary surgery? Ophthalmic Surgery 26(2): 108-110; 1995. 
15. Sherwood MB, Migdal CS, Hitchings RA, et al. Initial treatment of glaucoma: surgery or medications. Survey of Ophthalmology 37(4): 293-305; 1993.

16. Bartlett JD, Jaanus SD, eds. Clinical Ocular Pharmacology. 2nd ed. Boston: Butterworths; 1989: 245-265.

17. Apple DJ, Naumann GOH. Pathology of the Eye. New York: Springer-Verlag; 1986: 771-790.

18. Becker B. The decline of aqueous secretion and outflow with age. American Journal of Ophthalmology 46: $731 ; 1958$.

19. Lee WR. Doyne Lecture. The pathology of the outflow system in primary and secondary glaucoma. Eye 9(1): 1-23; 1995.

20. Schappert-Kimmijser J. A five-year follow-up of subjects with intraocular pressure of 22-30 $\mathrm{mm} \mathrm{Hg}$ without anomalies of optic nerve and visual field typical for glaucoma at first investigation. Ophthalmologica 162(4): 289-295; 1971.

21. Armaly MF, Krueger DE, Maunder L, et al. Biostatistical analysis of the collaborative glaucoma study: summary report of the risk factors for glaucomatous visual-field defects. Archives of Ophthalmology 98(12): 2163-2171; 1980.

22. Fechter RD, Weinreb RN. Mechanisms of optic nerve damage in primary open-angle glaucoma. Survey of Ophthalmology 39(1): 23-42; 1994.

23. Weinreb RN. Why study the ocular microcirculation in glaucoma. Journal of Glaucoma 1: 145-147; 1992.

24. Drance SM. Bowman Lecture. Glaucoma: Changing concepts. Eye 6(2): 337-345; 1992.

25. Jay JL. Rational choice of therapy in primary open-angle glaucoma. Eye 6(3): 243-247; 1992.

26. Brown MM, Brown GC, Spaeth GL. Improper topical self-administration of ocular medication among patients with glaucoma. Canadian Journal of Ophthalmology 19(1): 2-5; 1984.

27. Novack GD, David R, Lee PF, et al. Effect of changing medication regimens in glaucoma patients. Ophthalmologica 196(1): 23-28; 1988.

28. Frishman WH, Fuksbrumer MS, Tannenbaum M. Topical ophthalmic beta-adrenergic blockade for the treatment of glaucoma and ocular hypertension. Journal of Clinical Pharmacology 34(8): 795-803; 1994.

29. Zimmerman TJ, Kooner KS, editors. Ophtalmology Clinics of North America. New York, NY: WB Saunders and Co., 1989, 2(1): 77-87.

30. Trope GE, Menon IA, Liu GS, et al. Ocular timolol levels after drug withdrawal: an experimental model. Canadian Journal of Ophthalmology 29(5): 217-219; 1994.

31. Neufeld AH, Drance SM, VanBuskirk, eds. Pharmacology of Glaucoma. Williams and Wilkins; 1992, 6667, 293, 302-325.

32. Schoene RB, Martin TR, Charan NB, French CL. Timolol-induced bronchospasm in asthmatic bronchitis. Journal of the American Medical Association 245(14): 1460-1461; 1981.

33. O'Donoghue E. Beta-blockers and the elderly with glaucoma: are we adding insult to injury? British 
Journal of Ophthalmology 79(9): 794-796; 1995.

34. Brooks AM, Gillies WE. Ocular beta-blockers in glaucoma management: clinical pharmacological aspects. Drugs \& Aging 2(3): 208-221; 1992.

35. Axelsson U, Holmberg A. The frequency of cataract after miotic therapy. Acta Ophthalmologica 44(3): 421-429; 1966.

36. Hurvitz LM, Kaufman PL, Robin AL, et al. New developments in the drug treatment of glaucoma. Drugs 41(4): 514-532; 1991.

37. Chiou GC. Treatment of open-angle glaucoma and iscemic retinopathy with dopamine antagonists. Journal of Ocular Pharmacology 10(1): 371-377; 1994.

38. Crick RP, Newson RB, Shipley MJ, et al. The progress of the visual field in chronic simple glaucoma and ocular hypertension treated topically with pilocarpine or with timolol. Eye 4(4): 563-571; 1990.

39. Hong SJ, Chiou GC. Effects of dopamine agonists and antagonists on pulsatile blood flow of ocular hypertensive rabbits. Journal or Ocular Pharmacology 9(2): 117-124; 1993.

40. Chiou GC, Chen YJ. Effects of dopamine agonist, bromocriptine, and some dopamine antagonists on ocular blood flow. Journal of Ocular Pharmacology 8(4): 285-294; 1992.

41. Netland PA, Chaturvedi N, Dreyer EB. Calcium-channel blockers in the management of low-tension and open-angle glaucoma. American Journal of Ophthalmology 115(5): 608-613; 1993.

42. Gaspar AZ, Flammer J, Hendrickson P. Influence of nifedipine on the visual fields of patients with opticnerve-head diseases. European Journal of Ophthalmology 4(1): 24-28; 1994.

43. Schulzer M, Drance SM, Carter CJ, et al. Biostatistical evidence for two distinct chronic open-angle glaucoma populations. British Journal of Ophthalmology 74(4): 196-200; 1990.

44. Alm A, Widengard I, Kjellgren D, et al. Latanoprost administered once daily caused a maintained reduction of intraocular pressure in glaucoma patients treated concomitantly with timolol. British Journal of Ophthalmology 79(1): 12-16; 1995.

45. Bodor N. Designing safer ophthalmic drugs by soft drug approaches. Journal of Ocular Pharmacology 10(1): 3-15; 1994.

\section{BIOGRAPHY}

Kevin A. Waschke received his B.Sc. in biochemistry from McGill University (Montreal, Quebec, Canada) in 1994. He is presently a second-year medical student at McGill University. His work on the pharmacology of glaucoma was conducted during his first year of medical education at McGill.

Copyright (C) 1996 by MJM 